\title{
Moving Beyond Serendipity to Mechanism-Driven Psychiatric Therapeutics
}

\author{
Andrew A. Pieper ${ }^{1}$. Jay M. Baraban ${ }^{2}$
}

Published online: 26 June 2017

(C) The American Society for Experimental NeuroTherapeutics, Inc. 2017

The accidental discoveries of chlorpromazine, imipramine, and lithium as effective treatments for schizophrenia, depression, and bipolar disorder, respectively, revolutionized psychiatry in the early 1950s. This burst of serendipity transformed patient care and also gave birth to the notion that these mysterious diseases of the mind were due to a "chemical imbalance", thereby spawning the field of biological psychiatry. While these new drugs produced miraculous results compared with previous treatments, their shortcomings prompted research into developing new variations with superior efficacy and improved side effect profiles. Although tremendous progress has been made since the 1950s, currently available treatments are still far from ideal. Indeed, in 2017 a substantial number of patients display only partial or even no response to first- or second-line treatments for a range of psychiatric disorders. Thus, there is a pressing need for new treatment strategies that fundamentally improve the lives of patients.

In recent decades, unprecedented progress in understanding basic neuroscience, brain imaging, and psychiatric genomics has fostered optimism that scientific discovery will ultimately be translated into new and superior pharmacologic treatments for patients suffer-

Andrew A. Pieper

andrewapieper@gmail.com

Jay M. Baraban

jay.baraban@gmail.com

1 Department of Psychiatry, University of Iowa Carver College of Medicine, Iowa City, IA, USA

2 Solomon H. Snyder Department of Neuroscience and Department of Psychiatry and Behavioral Sciences, Johns Hopkins School of Medicine, Baltimore, MD, USA ing from mental illness. Similarly, the advent of rigorous, evidence-based psychotherapy also offers promise for revolutionizing patient care. However, new treatment regimens based on these breakthroughs have been slow to surface in the clinic, raising concerns about whether psychiatry is ready to move beyond serendipity and leverage scientific advances into improved treatments. Can psychiatry change from a discipline that has historically revolved around derivative optimization of serendipitously discovered treatments into one in which treatments emanate from deciphering the underlying pathophysiology of mental illness? Are there new treatment paradigms on the horizon, or are we meandering in the desert with the promised land of rationally designed treatments still decades away?

These questions provided the impetus for this special issue of Neurotherapeutics, 'Emerging Therapeutic Strategies in Psychiatry'. The articles contained in this issue provide a behind-the-scenes view from well-recognized leaders in the field of how this paradigm shift is progressing. Though progress is uneven, we hope you will agree that there is substantial basis for optimism.

In keeping with the spirit of a new paradigm, we have categorized the articles in this issue based on the approaches driving progress in the area being discussed, rather than organizing them using a conventional disease-based system. For example, articles grouped under 'Psychiatric Genomics' or 'Animal Models of Psychiatric Disease' emphasize the role of these approaches in recent advances. In addition, articles covering renewed interest in drugs discovered decades ago by serendipity are presented in the section entitled ' $\mathrm{Re}$ Emergence of Classic Psychiatric Drugs'. Finally, while approaches grounded in genetics and molecular pharmacology have attracted considerable attention, it is also important to 
highlight excitement surrounding innovative approaches in 'Psychotherapy'.

This issue begins with the 'Psychotherapy' section. Despite its long and rich history in mental health, many in the field have recently criticized psychotherapy as not being based on scientific evidence, or for being overly demanding on the limited time and finances of most patients. The utility of psychotherapy, however, crosses all forms of human illness, and dedicated clinicians in the field have clearly heard these criticisms and responded accordingly. In the first article of this section, Cook et al. [1] summarize the current state of evidence-based psychotherapy, dedicated to the mission of improving patient care and mitigating potential barriers to successful psychotherapy. Importantly, this approach is being implemented while preserving a crucial focus on the unique values and goals of patients. These authors provide an inspiring account of how rigorous evidence-based psychotherapy can be implemented in a clinical setting to improve both the efficacy and costeffectiveness of treating patients.

It is well known that medical and psychiatric diseases frequently occur together, compounding their severity and the challenges of treating both effectively. In the second article of the "Psychotherapy" section, Dindo et al. [2] describe a recently developed and empirically derived form of psychotherapy known as acceptance and commitment therapy (ACT). ACT is a transdiagnostic psychotherapeutic intervention that addresses both the mental and physical aspects of patient health with a unified model of behavior change. ACT rests on the premise that certain "negative" experiences, such as disappointment and anxiety, are inevitable features of human life. The aim of ACT is thus to help individuals develop the emotional skills or "psychological flexibility" needed to respond to challenging situations, rather than engage in counter-productive attempts to eliminate or suppress undesirable experiences. Psychological flexibility is strengthened with identification and committed pursuit of individually valued life areas, even when it may be difficult. This remarkably effective form of psychotherapy can be rapidly and affordably delivered to patients in a variety of formats, including 1-day workshops or tele-health set-ups, across a variety of mental health, primary care, and specialty medical clinics.

In the last article of the "Psychotherapy" section, MaplesKeller et al. [3] discuss how using 3-dimensional virtual reality technology to simulate traumatic experiences is revolutionizing traditional extinction-learning approaches for treating patients suffering from anxiety-producing conditioned fear responses. The efficacy of this novel approach has been rigorously established and carries clear applicability to treating the enormous number of patients suffering from phobias and post-traumatic stress.

The next section highlights progress in developing new diagnostic and therapeutic approaches being driven by insights gleaned from psychiatric genomics. Given the complexity of this topic, the first article in this section, by Jacob Michaelson [4], provides an accessible overview of the field along with in-depth discussion of the application of genomics to studying the etiology of neurodevelopmental disorders. The second article in this section, by Benjamin Pickard [5], describes modern genomic approaches that are being used to identify and explore key differences between patients that do or do not respond to lithium. This line of research provides one of several examples in this issue of how new approaches are being used to improve classic treatments that were originally based on serendipitous discovery. The insights described by Pickard will hopefully soon enable clinicians to accurately predict which patients are likely to respond favorably to the mood-stabilizing effects of lithium. The next article in this section, by Kabir et al. [6], discusses efforts to capitalize on the finding that mutations in genes encoding calcium channel subunits are associated with an unusually wide variety of psychiatric disorders. The availability of various calcium channel modulators and transgenic mouse lines with altered expression of implicated subunits makes this a rich area of investigation. Kabir et al. [6] review clinical studies that have examined efficacy of these agents in patients with bipolar disorder, schizophrenia, and drug addiction, as well as animal laboratory studies that have identified cellular events that underlie behavioral correlates of human disorders. The next article in the "Psychiatric Genomics" section, by Michael Lutter [7], shows how multiple approaches are being used to make inroads on treating very challenging patients suffering from eating disorders. Leads from advances in identifying endocrine factors controlling feeding and satiety are being integrated with clinical trials of agents already approved for safe use in other disorders. Furthermore, pedigree analysis has yielded identification of 2 unique genetic mutations that segregate with patients afflicted with eating disorders. Just as identification of rare familial mutations associated with Parkinson's disease has paved the way to mapping a common pathophysiology relevant to sporadic forms of the disease, the seminal findings described by Lutter [7] appear to provide a secure foothold for unraveling the pathophysiology of eating disorders with the help of transgenic mice bearing these mutations. In the last article in the "Psychiatric Genomics" section, Tomoda et al. [8] provide an overview of recent advances examining the role of disrupted in schizophrenia 1 (DISC1) in a complex network of cellular signaling pathways. From the initial identification of DISC1 as the site of a translocation linked to psychotic and affective disorders, investigators have uncovered a bewildering array of DISC1 interactors, highlighting its central role in multiple cellular functions. Thus, Tomoda et al. [8] discuss innovative approaches needed to 
decipher which of DISC1's pleiotropic effects are linked to psychiatric disease and represent potential therapeutic targets.

Several common themes are shared by the articles in the section on "Animal Models of Psychiatric Disease". For example, 3 articles address different aspects of hippocampal circuitry. Articles by Sahay and McAvoy [9], and Drew and Huckleberry [10] discuss the rapid progress in understanding the role of adult hippocampal neurogenesis in cognitive and emotional processing, information that is likely to shape new therapeutic strategies based on manipulating neurogenesis. In addition, Haberman et al. [11] focus on recent studies indicating that hyperactivity of hippocampal circuitry contributes to cognitive decline with aging, and that agents that suppress this hyperactivity show translational potential. In a similar fashion, articles by Han and Nestler [12], and Cooper et al. [13] discuss how advances in understanding reward circuitry and its response to chronic stress have yielded exciting new leads for therapeutic targets for treating depression and drug addiction. In the next article, $\mathrm{Ku}$ and Han [14] detail recent advances using animal models to suggest that pharmacologic modulation of hyperpolarization-activated cyclic nucleotide-gated ion channels holds promise as a novel antidepressant treatment strategy. Lastly, the article on vagal nerve stimulation by Carreno and Frazer [15] illustrates how both animal model testing and human brain imaging can be used together to validate and improve this new treatment strategy for patients suffering from severe major depression. Furthermore, it is important to point out that other forms of brain stimulation, including deep brain stimulation, transcranial direct current stimulation, and transcranial magnetic stimulation, are also under active investigation and have been reviewed extensively elsewhere.

Although there is a tremendous effort to move beyond serendipity in developing novel treatment strategies, it would be foolish not to take advantage of the rich history of serendipity in psychiatry. Thus, several articles in the final section, entitled "Re-emergence of Classic Psychiatric Drugs", demonstrate that this line of research is alive and well. The unexpected discovery that ketamine can rapidly elicit robust antidepressant effects has energized efforts to understand its mode of action and the pathophysiology of depression. Harraz and Snyder [16] present an overview of recent work in this rapidly moving field, including identification of new downstream events that may provide future targets for therapeutic intervention. Furthermore, after a long hiatus, psilocybin, the active agent in psychedelic "magic mushrooms" that was identified in the 1950s, has re-emerged as a potential therapeutic agent in anxiety and addictive disorders, as described by Johnson and Griffiths [17]. Psilocybin is closely related to the serendipitous discovery of the psychedelic properties of lysergic acid diethylamide (LSD) by Albert Hofman in 1938, since he also spearheaded efforts to identify the active compound in magic mushrooms. As the structure of psilocybin is nearly identical to that of serotonin, this discovery launched efforts to determine whether serotonin, which had been originally discovered as a pressor substance in serum, was also a neurotransmitter in the brain. Indeed, this effort proved to be one of the foundations of modern neuroscience and psychiatry. Thus, it is fascinating that psilocybin itself may have direct therapeutic value, in addition to being indirectly responsible for the discovery of the serotonin system in the brain, and hence the introduction of selective serotonin reuptake inhibitors widely prescribed to treat patients with depression and anxiety. Furthermore, 3,4-methylenedioxy-methamphetamine (MDMA), a synthetic psychedelic agent, has been found to promote social intimacy and, as described by Sessa [18], is showing promise in a variety of clinical contexts.

Last, but not least, Nucifora et al. [19] discuss the current status of research on clozapine, the first atypical antipsychotic, discovered in 1958. Although pharmaceutical companies have tried for decades to unlock the secrets of its superior efficacy, the search for this Holy Grail is ongoing. Hopefully, invigorating classic pharmacological approaches with a modern armamentarium of genomics, imaging, and transgenic mice will lead to success in the near future.

As amply illustrated by the articles in this special issue, psychiatry is moving away from relying on serendipity and towards exploiting new scientific approaches to identify novel therapeutics. There are early indications that this process will pay off, but given the complexities inherent in understanding the brain and mental illness it is also reasonable to expect the road to be long. We hope you will enjoy reading these updates about how this paradigm shift is proceeding.

We want to take this opportunity to thank all of the contributors and reviewers for their outstanding work. In addition, our sincere thanks go to Linda Powell for expertly shepherding this volume through the publishing process.

\section{References}

1. Cook SC, Schwartz AC, Kaslow NJ. Evidence-Based Psychotherapy. Neurotherapeutics (this issue). doi: 10.1007/ s13311-017-0549-4

2. Dindo L, Van Liew J, and Arch J. Acceptance and Commitment Therapy: A Transdiagnostic Behavioral Intervention for Mental Health and Medical Conditions. Neurotherapeutics 2017 (this issue). doi: 10.1007/s13311-017-0521-3

3. Maples-Keller JL, Yasinski C, Manjin N, Rothbaum BO. Virtual reality-enhanced extinction of phobias and post-traumatic stress. Neurotherapeutics 2017 (this issue). doi: 10.1007/s13311-0170534-y

4. Michaelson J. Genetic Approaches to Understanding Psychiatric Disease. Neurotherapeutics 2017 (this issue). doi: 10.1007/ s13311-017-0551-x

5. Pickard B. Genomics of Lithium Action and Response. Neurotherapeutics 2017 (this issue). doi: 10.1007/s13311-0170554-7 
6. Kabir ZD, Martinez-Rivera A, Rajadhyaksha AM. From gene to behavior: L-type calcium channel mechanisms underlying neuropsychiatric symptoms. Neurotherapeutics 2017 (this issue). doi: 10. 1007/s13311-017-0532-0

7. Lutter M. Emerging treatments in eating disorders. Neurotherapeutics 2017 (this issue). doi: 10.1007/s13311-017-0535-x

8. Tomoda T. Role of DISC1 in neuronal trafficking and its implication in neuropsychiatric manifestation and neurotherapeutics.Neurotherapeutics 2017 (this issue). doi: 10 . 1007/s13311-017-0556-5

9. Sahay A. and McAvoy KM. Targeting Adult Neurogenesis to Optimize Hippocampal Circuits in Aging. Neurotherapeutics 2017 (this issue). doi:10.1007/s13311-017-0539-6

10. Drew M. and Huckleberry KA. Modulation of Aversive Memory by Adult Hippocampal Neurogenesis. Neurotherapeutics 2017 (this issue). doi:10.1007/s13311-017-0528-9

11. Haberman R, Branch A., and Gallagher M. Targeting Neural Hyperactivity as a Treatment to Stem Progression of Late-Onset Alzheimer's Disease.Neurotherapeutics 2017 (this issue). doi:10.1007/s13311-017-0552-9

12. Han MH and Nestler E. Neural Substrates of Depression and Resilience. Neurotherapeutics 2017 (this issue). doi:10.1007/ s13311-017-0527-x
13. Cooper S, Robinson AJ, and Mazei-Robinson M. Reward Circuitry in Addiction. Neurotherapeutics 2017 (this issue). doi:10.1007/ s13311-017-0525-Z

$14 \mathrm{Ku} \mathrm{SM}$ and Han MH. HCN Channel Targets for Novel Antidepressant Treatment. Neurotherapeutics 2017 (this issue). doi:10.1007/s13311-017-0538-7

15. Carreno FR and Frazer A. Vagal Nerve Stimulation for Treatment Resistant Depression. Neurotherapeutics 2017 (this issue). doi:10.1007/s13311-017-0537-8

16. Harraz MM and Snyder SH. Antidepressant Actions of Ketamine Mediated by mTOR, nitric oxide and Rheb. Neurotherapeutics 2017 (this issue). doi:10.1007/s13311-017-0540-0

17. Johnson MW and Griffiths RR. Potential Therapeutic Effects of Psilocybin. Neurotherapeutics 2017 (this issue). doi:10.1007/ s13311-017-0542-y

18. Sessa B. Why Psychiatry Needs MDMA: A Child Psychiatrist's Perspective. Neurotherapeutics 2017 (this issue). doi:10.1007/ s13311-017-0531-1

19. Nucifora FC Jr., Mihaljevic M, Lee BJ, and Sawa A. Clozapine: Beyond Neuroreceptor Occupancy. Neurotherapeutics 2017 (this issue). doi:10.1007/s13311-017-0552-9 